\title{
Multi-messenger searches via lceCube's high-energy neutrinos and gravitational-wave detections of LIGO/Virgo
}

\section{The IceCube Collaboration}

(a complete list of authors can be found at the end of the proceedings)

E-mail:dv2397@columbia.edu

We summarize initial results for high-energy neutrino counterpart searches coinciding with gravitational-wave events in LIGO/Virgo's GWTC-2 catalog using IceCube's neutrino triggers. We did not find any statistically significant high-energy neutrino counterpart and derived upper limits on the time-integrated neutrino emission on Earth as well as the isotropic equivalent energy emitted in high-energy neutrinos for each event.

Corresponding authors: Doğa Veske ${ }^{1 *}$, Raamis Hussain ${ }^{2}$, Zsuzsa Márka $^{1}$, Stefan Countryman ${ }^{1}$, Alex Pizzuto $^{2}$, Yasmeen Asali ${ }^{1,3}$, Ana Silva Oliveira ${ }^{1}$ and Justin Vandenbroucke ${ }^{2}$

${ }^{1}$ Columbia University, NY, USA

${ }^{2}$ University of Wisconsin Madison, WI, USA

${ }^{3}$ Yale University, CT, USA

${ }^{*}$ Presenter

$37^{\text {th }}$ International Cosmic Ray Conference (ICRC 2021)

July 12th - 23rd, 2021

Online - Berlin, Germany 


\section{Introduction}

Astrophysics presents us the opportunity to observe physical phenomena which are not feasibly created via experiments on Earth. Observing such astrophysical events with multiple messengers can extend our understanding by showing a more complete picture of the emission processes from the astrophysical source. With developing detectors, platforms for communication between astronomy communities, and efficient statistical methods [1], multi-messenger searches and detections [24] have become a reality. One multi-messenger combination that hasn't been observed yet is of gravitational-waves (GWs) and high-energy neutrinos, despite previous searches [5-14]. Here we present our searches for high-energy neutrinos originating from the sources of GW events in the GWTC-2 catalog of the LIGO Scientific and Virgo Collaborations [15] with the triggers of the IceCube detector [16]. The methodology of the searches are summarized in Sec. 2 and the results are given in Sec. 3. Sec. 4 has the conclusion.

\subsection{First half of the third observing run of advanced LIGO and Virgo}

First half of the third observing run of advanced LIGO [17] and Virgo [18] detectors, which is generally referred as O3a, started on April $1^{\text {st }}, 2019$ and ended six months later on October $1^{\text {st }}$. It was a combined run of the two LIGO detectors in the US (Hanford and Livingston) and the Virgo detector in Italy. During the run, candidate events were announced through Gravitationalwave Candidate Event Database (GraceDB) ${ }^{1}$ in low-latency. The IceCube Collaboration performed realtime follow-up of these open public alerts [19-21] and sent out Gamma-ray Coordinates Network circulars for our findings. After the offline analysis by LIGO Scientific and Virgo Collaborations, the second gravitational-wave transients catalog GWTC-2 was released with total of 39 events of coalescing binary compact objects from O3a [15]. Twenty-six of these events were previously reported in low-latency during $\mathrm{O} 3 \mathrm{a}$, while thirteen events were new detections found in the offline analysis of the $\mathrm{O} 3 \mathrm{a}$ data.

\section{Searches}

There are two pipelines used for the searches; Low Latency Algorithm for Multi-messenger Astrophysics (LLAMA) and the Unbinned Maximum Likelihood (UML). Although both pipelines are essentially looking for the same thing, there are differences between them. Each search uses a different statistical approach to search for and quantify the significance of IceCube neutrinos coincident with compact binary mergers. We briefly describe both methods below. For details see Ref. [22] for the LLAMA search method and Ref. [21] for the UML method. Both pipelines use the gamma-ray follow up (GFU) stream provided by IceCube [23] for high-energy neutrino data. This data sample mostly consists of atmospheric muons with a small portion of astrophysical neutrino triggers.

\subsection{Low Latency Algorithm for Multi-messenger Astrophysics}

The LLAMA search calculates the Bayesian probabilities of different hypotheses arising from the combinations of GW or neutrinos being astrophysically related or not, considering the scenario

\footnotetext{
${ }^{1}$ https://gracedb.ligo.org
} 
of them being not astrophysical at all as well. The odds ratio of the GW and neutrino messengers being astrophysically related compared to all the other combinations is used as the test statistic. The priors on the probabilities are either obtained from the detectors' background trigger rate due to noise or by assuming an astrophysical energy emission distribution for GW and neutrinos. In the case for certain GW detections, as in here, likelihoods for messengers' origin (noise or astrophysical) and their relation are calculated by using their detection times, sky localizations, reconstructed energy of neutrinos and the estimated luminosity distance of the GW event. Considering the pipelines here, using the distance of the GW event is unique to the LLAMA search which is used to account for the propagation of the neutrinos in space. The maximum allowed time difference between related GW and neutrinos is $\pm 500 \mathrm{~s}$ [24] and temporally closer detections are favored.

The significances are obtained by using precomputed background distributions which contain the results of randomly matched neutrinos and simulated GWs at the detection rate of the GFU stream. For each type of merger (binary black hole (BBH), neutron star black hole (NSBH) or binary neutron star (BNS)) different background distributions are obtained. The reason for this is the different detection horizon of the GW detectors for each source type due to the different signal power created by them in the detectors. This produces different distance distributions for each type of merger which affects the significance since the distance information is used in the test statistic calculation.

\subsection{Unbinned Maximum Likelihood}

This method uses an unbinned maximum likelihood (UML) which is weighted by a spatial weight derived from the sky localization of the GWs. The method is briefly described here, but for full details on the method, see [14].

Firstly, the sky is divided into equal area bins using the Healpix pixelization scheme. The test statistic is then calculated in every pixel by maximizing the log-likelihood ratio with respect to the number of signal events, $n_{s}$, and the spectral index of the source, $\gamma$. The test statistic in each pixel is then weighted by the spatial weight which describes the probability of the GW source being located in the given pixel. The maximum test statistic in the sky is chosen as the best fit location for the scan.

To compute the significance of a given observation, we run 30,000 trials for each GW event with scrambled neutrino data to build a background test statistic distribution. We then compare the observed test statistic to the background distribution to compute a p-value for each GW.

Two analyses are performed using the UML method. The first is a short timescale follow up of every reported GW event. Here we search for neutrinos within a $\pm 500 \mathrm{~s}$ time window centered around the GW merger time. The goal of this analysis is to search for prompt neutrino emission just before and just after the GW merger. This analysis was run in real-time during the $\mathrm{O} 3$ observing run and responded to all public alerts sent by LVC in low-latency.

The second analysis is a longer time scale search targeting all binary neutron star and neutron star-black hole mergers. Here we search a $[-0.1,+14]$ day time window around the GW merger time. This search is motivated by several models which predict neutrino emission specifically from BNS and NSBH mergers on longer time scales [25, 26]. This analysis was run on three events from GWTC-2: GW190425, GW190426_152155, and GW190814 which contain at least one compact object less massive than $3 \mathrm{M}_{\odot}$ which could be a neutron star. 


\section{Results}

Among the events in GWTC-2 no significant neutrino emission was observed in either the UML or LLAMA analyses. The long time scale searches with the UML also yielded no significant results. Table 1 shows the full results for the $1000 \mathrm{~s}$ follow up of the events from the GWTC-2 catalog. The table contains the $90 \%$ confidence level upper limits (UL) computed for the energy scaled time-integrated neutrino flux $\left(E^{2} F\right)$ and the isotropic equivalent energy $\left(E_{\text {iso }}\right)$ emitted in high-energy neutrinos from each GW event. Table 2 shows the results for the 2 week follow up of BNS/NSBH candidates from GWTC-2. Fig. 2 shows the $\mathrm{E}_{\text {iso }}$ upper limits as a function of the distance to the source including the events from GWTC-1 [27] as well. The overlay of the neutrino and the zoomed in GW sky localization for the most significant event GW190728_064510 which has a $p$-value of $1.3 \%$ and $4 \%$ in the LLAMA and UML pipelines respectively is shown in Fig. 1. The candidate event of GW190728_064510 (S190728q) was also the only event that had $\leq 1 \%$ $p$-value in at least one of the two analyses during the realtime follow-up. The coordinates for the signficant neutrino were shared via GCN circular ${ }^{2}$. The coincident neutrino arrived $360 \mathrm{~s}$ before the GW merger and had a reconstructed energy of $601 \mathrm{GeV}$. No additional counterpart was found from other observatories (i.e. Ref. [28]).

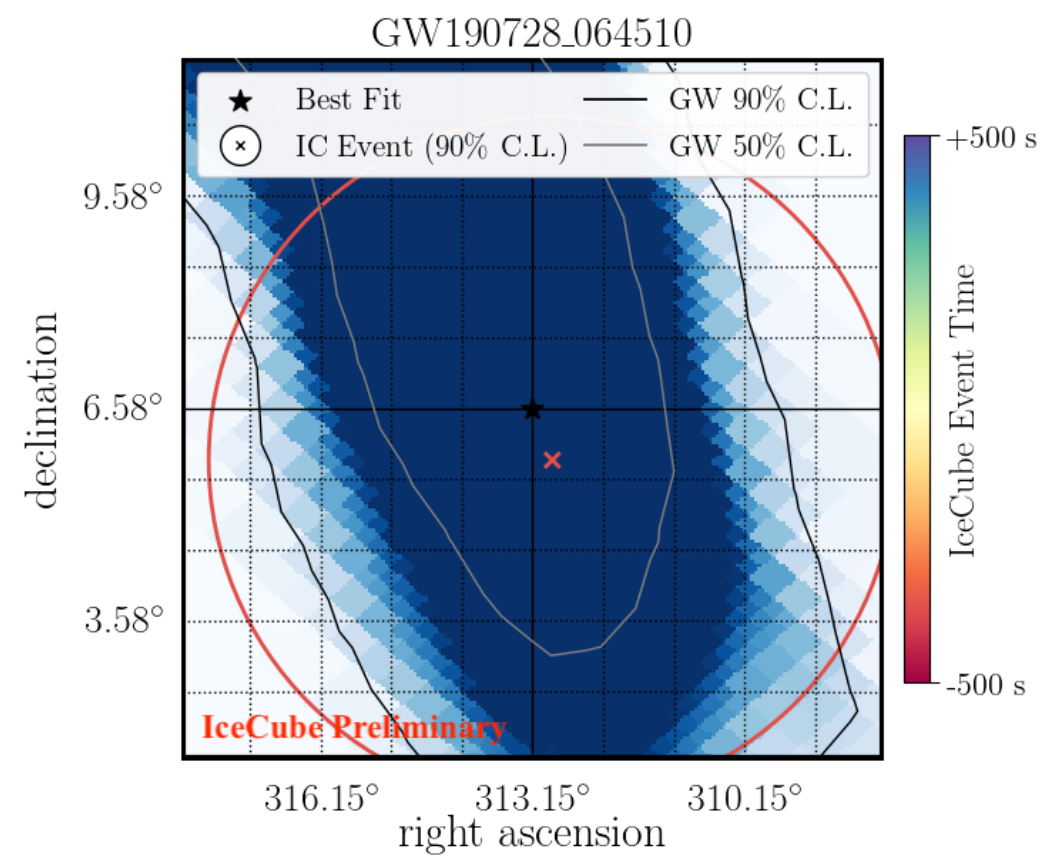

Figure 1: The zoomed in display of the sky localization of the most significant high-energy neutrino-GW pair in equatorial coordinates. The blue gradient represents the sky localization probability of the GW with the darker color representing higher probability. The red cross represents the best fit direction for the coincident neutrino with the circle representing the $90 \%$ containment angular error region. The figure is centered around the best fit location of the UML search, which is shown with a black star at the center.

\footnotetext{
${ }^{2}$ https://gcn.gsfc.nasa.gov/gcn3/25210.gcn3
} 


\begin{tabular}{|c|c|c|c|c|c|c|}
\hline & & \multicolumn{2}{|c|}{ LLAMA } & \multicolumn{3}{|c|}{ UML } \\
\hline Event & Type & $p$-value & $\begin{array}{c}\mathrm{E}^{2} \mathrm{~F} \text { UL } \\
{\left[\mathrm{GeVcm}^{-2}\right]}\end{array}$ & $p$-value & $\begin{array}{c}\mathrm{E}^{2} \mathrm{~F} \mathrm{UL} \\
{\left[\mathrm{GeVcm}^{-2}\right]}\end{array}$ & $\mathrm{E}_{\text {iso }}$ UL [erg] \\
\hline GW190408_181802 & $\mathrm{BBH}$ & 0.16 & 0.048 & 0.17 & 0.0512 & $4.85 \times 10^{53}$ \\
\hline GW190412 & $\mathrm{BBH}$ & 0.19 & 0.041 & 0.13 & 0.0459 & $8.31 \times 10^{52}$ \\
\hline GW190413_052954 & $\mathrm{BBH}$ & 0.21 & 0.087 & 0.28 & 0.133 & $7.01 \times 10^{54}$ \\
\hline GW190413_134308 & $\mathrm{BBH}$ & 0.18 & 0.34 & 0.34 & 0.270 & $2.84 \times 10^{55}$ \\
\hline GW190421_213856 & $\mathrm{BBH}$ & 0.77 & 0.46 & 0.56 & 0.393 & $1.40 \times 10^{55}$ \\
\hline GW190424_180648 & $\mathrm{BBH}$ & 0.58 & 0.32 & 0.23 & 0.233 & $5.37 \times 10^{54}$ \\
\hline GW190425 & BNS & 0.16 & 0.22 & 0.94 & 0.176 & $1.66 \times 10^{52}$ \\
\hline GW190426_152155 & NSBH & 0.12 & 0.082 & 0.12 & 0.0942 & $5.65 \times 10^{52}$ \\
\hline GW190503_185404 & $\mathrm{BBH}$ & 0.87 & 0.54 & 0.34 & 0.584 & $4.99 \times 10^{54}$ \\
\hline GW190512_180714 & $\mathrm{BBH}$ & 0.67 & 0.23 & 0.85 & 0.199 & $1.74 \times 10^{54}$ \\
\hline GW190513_205428 & $\mathrm{BBH}$ & 0.97 & 0.043 & 0.94 & 0.0514 & $6.73 \times 10^{53}$ \\
\hline GW190514_065416 & $\mathrm{BBH}$ & 0.28 & 0.089 & 0.44 & 0.0453 & $3.96 \times 10^{54}$ \\
\hline GW190517_055101 & $\mathrm{BBH}$ & 0.14 & 0.48 & 0.26 & 0.366 & $6.05 \times 10^{54}$ \\
\hline GW190519_153544 & $\mathrm{BBH}$ & 0.063 & 0.15 & 0.21 & 0.0914 & $3.20 \times 10^{54}$ \\
\hline GW190521 & $\mathrm{BBH}$ & 0.47 & 0.37 & 0.63 & 0.359 & $1.90 \times 10^{55}$ \\
\hline GW190521_074359 & $\mathrm{BBH}$ & 0.16 & 0.049 & 0.15 & 0.0451 & $2.36 \times 10^{53}$ \\
\hline GW190527_092055 & $\mathrm{BBH}$ & 0.61 & 0.41 & 0.88 & 0.326 & $1.01 \times 10^{55}$ \\
\hline GW190602_175927 & $\mathrm{BBH}$ & 0.22 & 0.34 & 0.17 & 0.370 & $9.73 \times 10^{54}$ \\
\hline GW190620_030421 & $\mathrm{BBH}$ & 0.15 & 0.36 & 0.23 & 0.121 & $4.13 \times 10^{54}$ \\
\hline GW190630_185205 & $\mathrm{BBH}$ & 0.38 & 0.15 & 0.81 & 0.427 & $5.31 \times 10^{53}$ \\
\hline GW190701_203306 & $\mathrm{BBH}$ & 1.0 & 0.039 & 0.87 & 0.0385 & $7.65 \times 10^{53}$ \\
\hline GW190706_222641 & $\mathrm{BBH}$ & 0.99 & 0.036 & 0.92 & 0.0356 & $3.17 \times 10^{54}$ \\
\hline GW190707_093326 & $\mathrm{BBH}$ & 0.43 & 0.24 & 0.63 & 0.202 & $4.74 \times 10^{53}$ \\
\hline GW190708_232457 & $\mathrm{BBH}$ & 0.11 & 0.11 & 0.56 & 0.0720 & $1.62 \times 10^{53}$ \\
\hline GW190719_215514 & $\mathrm{BBH}$ & 0.79 & 0.054 & 0.91 & 0.0512 & $4.90 \times 10^{54}$ \\
\hline GW190720_000836 & $\mathrm{BBH}$ & 0.98 & 0.13 & 0.94 & 0.0872 & $5.34 \times 10^{53}$ \\
\hline GW190727_060333 & $\mathrm{BBH}$ & 0.79 & 0.38 & 0.74 & 0.324 & $1.53 \times 10^{55}$ \\
\hline GW190728_064510 & $\mathrm{BBH}$ & 0.013 & 0.89 & 0.04 & 0.315 & $6.36 \times 10^{53}$ \\
\hline GW190731_140936 & $\mathrm{BBH}$ & 0.29 & 0.93 & 0.61 & 0.385 & $1.81 \times 10^{55}$ \\
\hline GW190803_022701 & $\mathrm{BBH}$ & 0.21 & 0.037 & 0.64 & 0.0354 & $1.69 \times 10^{54}$ \\
\hline GW190814 & $\mathrm{BBH}$ & 1.0 & 0.24 & 1.0 & 0.259 & $5.68 \times 10^{52}$ \\
\hline GW190828_063405 & $\mathrm{BBH}$ & 0.86 & 0.21 & 0.98 & 0.178 & $2.74 \times 10^{54}$ \\
\hline GW190828_065509 & $\mathrm{BBH}$ & 0.72 & 0.38 & 0.84 & 0.368 & $3.73 \times 10^{54}$ \\
\hline GW190909_114149 & $\mathrm{BBH}$ & 0.56 & 0.11 & 0.39 & 0.136 & $1.33 \times 10^{55}$ \\
\hline GW190910_112807 & $\mathrm{BBH}$ & 0.16 & 0.45 & 0.77 & 0.177 & $1.90 \times 10^{54}$ \\
\hline GW190915_235702 & $\mathrm{BBH}$ & 0.40 & 0.036 & 0.44 & 0.0354 & $3.61 \times 10^{53}$ \\
\hline GW190924_021846 & $\mathrm{BBH}$ & 0.038 & 0.037 & 0.23 & 0.0346 & $4.46 \times 10^{52}$ \\
\hline GW190929_012149 & $\mathrm{BBH}$ & 0.091 & 0.34 & 0.22 & 0.276 & - \\
\hline GW190930_133541 & $\mathrm{BBH}$ & 0.19 & 0.038 & 0.31 & 0.0427 & $1.05 \times 10^{53}$ \\
\hline
\end{tabular}

Table 1: Results for the events in GWTC-2 for the $1000 \mathrm{~s}$ follow up. GW190814 is labelled as a BBH merger here although the type of the lighter object at $\sim 2.6 \mathrm{M}_{\odot}$ is unknown [29]. Note that the $E_{\text {iso }} \mathrm{UL}$ for GW190929_012149 could not be properly calculated due to the distance measure being undefined for many pixels in the skymap, so it is omitted here. 


\begin{tabular}{|c|c|cc|}
\hline Event & Type & $p$-value & $\mathrm{E}^{2} \mathrm{~F} \mathrm{UL}\left[\mathrm{GeVcm}^{-2}\right]$ \\
\hline GW190425 & BNS & 0.43 & 0.661 \\
\hline GW190426_152155 & NSBH & 0.21 & 0.248 \\
\hline GW190814 & BBH & 0.59 & 0.309 \\
\hline
\end{tabular}

Table 2: Results for the 2 week follow up analysis using the UML method. These 3 events from GWTC-2 were followed up as they were the only potential BNS/NSBH candidates.

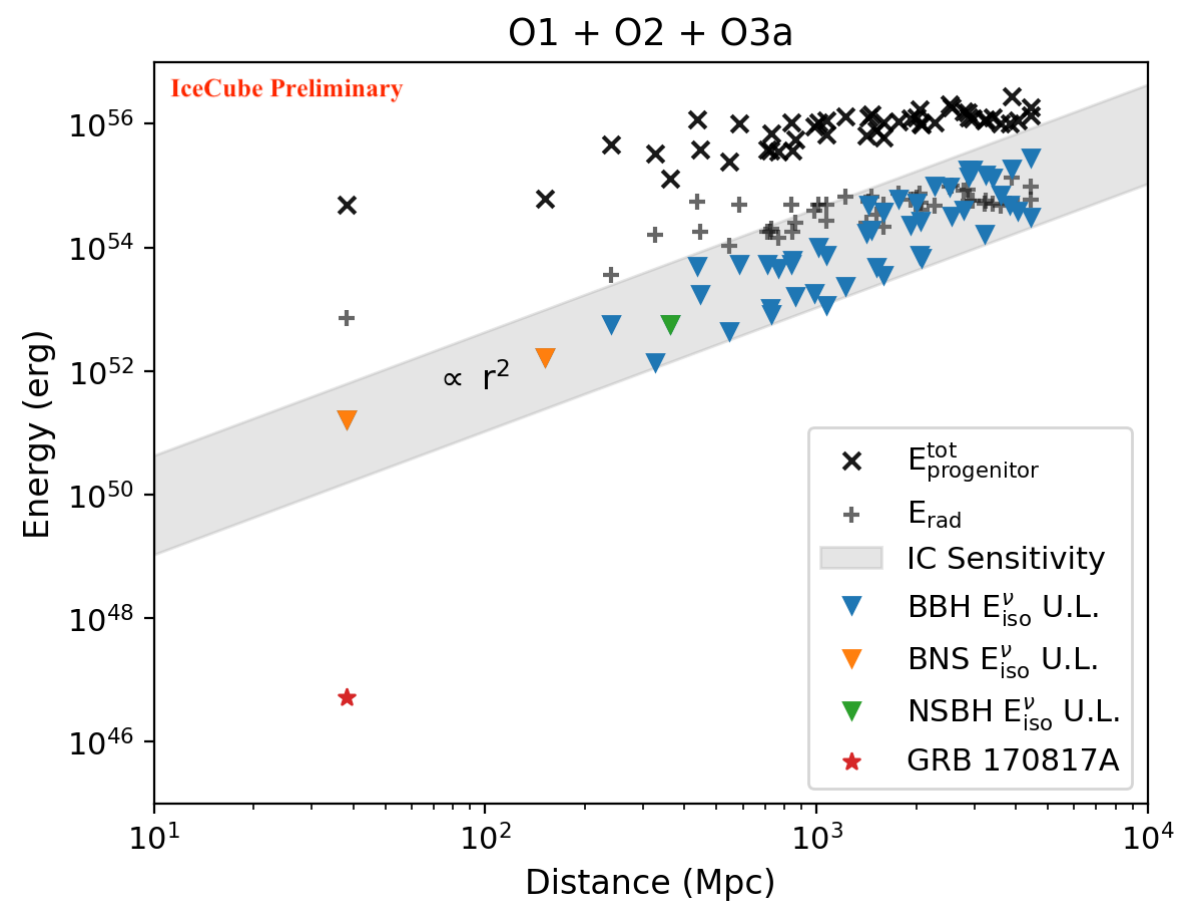

Figure 2: $90 \%$ UL on the isotropic equivalent energy emitted in high-energy neutrinos during a $1000 \mathrm{~s}$ time window (blue and orange triangles). $E_{\text {progenitor }}^{\text {tot }}$ (black cross) is the total rest mass energy of the progenitors and $E_{\text {rad }}$ (gray cross) is the total radiated energy of the binary system. While not all of the progenitor energy is available for acceleration processes, we show it here as a relevant energy scale in the binary system. The median distances for each GW are taken from GWTC-1 and GWTC-2 [15, 27]. Note that errors on the distance measurements are significant but not shown here for clarity. The red star shows the measured $E_{\text {iso }}$ for GRB 170817A by Fermi GBM taken from [3]. The gray band represents the range of $90 \% E_{\text {iso }}$ ULs that IceCube can set based on the range of point source sensitivities.

\section{Conclusion}

We summarized our searches for high-energy neutrino counterparts to GW events and their results for the events in the GWTC-2 catalog. Searches were done by two pipelines, LLAMA and UML, and neither of them found any statistically significant event. We also derived upper limits on the neutrino emission fluence on Earth and isotropically emitted energy in high-energy neutrinos. The searches for high-energy neutrino counterparts to GW events with the neutrino triggers of IceCube will continue as new GW catalogs are released. 


\section{Acknowledgements}

The Columbia Experimental Gravity group is grateful for the generous support of the National Science Foundation under grant PHY-2012035.

\section{References}

[1] D. Veske, Z. Márka, I. Bartos, and S. Márka The Astrophysical Journal 908 no. 2, (Feb, 2021) 216.

[2] B. P. Abbott et al. The Astrophysical Journal Letters 848 (Oct., 2017) L12.

[3] LIGO Scientific, Virgo, Fermi-GBM, INTEGRAL Collaboration, B. P. Abbott et al. Astrophys. J. 848 no. 2, (2017) L13.

[4] M. G. Aartsen et al. Science 361 (July, 2018) .

[5] Y. Aso et al. Class. Quantum Grav 25 no. 11, (June, 2008) 114039.

[6] V. van Elewyck et al. Int. J. Mod. Phys. D 18 (2009) 1655-1659.

[7] I. Bartos, C. Finley, A. Corsi, and S. Márka Phys.Rev.Lett. 107 no. 25, (Dec., 2011) 251101.

[8] B. Baret et al. Phys.Rev.D 85 no. 10, (May, 2012) 103004.

[9] S. Adrián-Martínez et al. JCAP 6 (June, 2013) 008.

[10] M. G. Aartsen et al. Phys.Rev.D 90 no. 10, (Nov., 2014) 102002.

[11] S. Adrián-Martínez et al. Phys.Rev.D 93 no. 12, (June, 2016) 122010.

[12] A. Albert et al. Astrophys.J.Lett. 850 (Dec., 2017) L35.

[13] A. Albert et al. Phys.Rev.D 96 no. 2, (July, 2017) 022005.

[14] IceCube Collaboration, M. G. Aartsen et al. Astrophys. J. Lett. 898 no. 1, (2020) L10.

[15] LIGO Scientific and Virgo Collaboration, R. Abbott et al. Phys. Rev. X 11 (Jun, 2021) 021053.

[16] IceCube Collaboration, M. G. Aartsen et al. JINST 12 no. 03, (2017) P03012.

[17] LIGO Scientific Collaboration, J. Aasi et al. Class. Quant. Grav. 32 (2015) 074001.

[18] Virgo Collaboration, F. Acernese et al. Class. Quant. Grav. 32 no. 2, (2015) 024001.

[19] S. Countryman, A. Keivani, I. Bartos, Z. Marka, T. Kintscher, R. Corley, E. Blaufuss, C. Finley, and S. Marka, "Low-latency algorithm for multi-messenger astrophysics (llama) with gravitational-wave and high-energy neutrino candidates," 2019.

https://arxiv. org/abs/1901.05486. 
[20] A. Keivani, D. Veske, S. Countryman, I. Bartos, K. R. Corely, Z. Marka, and S. Marka, "Multi-messenger Gravitational-Wave + High-Energy Neutrino Searches with LIGO, Virgo and IceCube," in Proceedings of 36th International Cosmic Ray Conference PoS(ICRC2019), vol. 358, p. 930. 2019. arXiv: 1908.04996 [astro-ph.HE].

[21] R. Hussain, J. Vandenbroucke, and J. Wood, "A Search for IceCube Neutrinos from the First 33 Detected Gravitational Wave Events," in Proceedings of 36th International Cosmic Ray Conference - PoS(ICRC2019), vol. 358, p. 918. 2019. arXiv: 1908.07706 [astro-ph.HE].

[22] I. Bartos, D. Veske, A. Keivani, Z. Márka, S. Countryman, E. Blaufuss, C. Finley, and S. Márka Phys. Rev. D 100 (Oct, 2019) 083017.

[23] IceCube Collaboration, T. Kintscher J. Phys. Conf. Ser. 718 no. 6, (2016) 062029.

[24] B. Baret, I. Bartos, B. Bouhou, A. Corsi, I. D. Palma, C. Donzaud, V. V. Elewyck, C. Finley, G. Jones, A. Kouchner, S. Márka, Z. Márka, L. Moscoso, E. Chassande-Mottin, M. A. Papa, T. Pradier, P. Raffai, J. Rollins, and P. Sutton Astroparticle Physics 35 no. 1, (2011) 1 - 7.

[25] K. Fang and B. D. Metzger Astrophys. J. 849 no. 2, (2017) 153.

[26] V. Decoene, C. Guépin, K. Fang, K. Kotera, and B. D. Metzger JCAP 04 (2020) 045.

[27] LIGO Scientific, Virgo Collaboration, B. P. Abbott et al. Phys. Rev. X 9 no. 3, (2019) 031040 .

[28] A. Keivani, J. A. Kennea, P. A. Evans, A. Tohuvavohu, R. Rapisura, S. R. Oates, S. Countryman, I. Bartos, Z. Márka, D. Veske, S. Márka, and D. B. Fox The Astrophysical Journal 909 no. 2, (Mar, 2021) 126.

[29] LIGO Scientific, Virgo Collaboration, R. Abbott et al. Astrophys. J. Lett. 896 no. 2, (2020) L44. 


\section{Full Author List: IceCube Collaboration}

R. Abbasi ${ }^{17}$, M. Ackermann ${ }^{59}$, J. Adams ${ }^{18}$, J. A. Aguilar ${ }^{12}$, M. Ahlers ${ }^{22}$, M. Ahrens ${ }^{50}$, C. Alispach ${ }^{28}$, A. A. Alves Jr. ${ }^{31}$, N. M. $\mathrm{Amin}^{42}$, R. An ${ }^{14}$, K. Andeen ${ }^{40}$, T. Anderson ${ }^{56}$, G. Anton ${ }^{26}$, C. Argüelles ${ }^{14}$, Y. Ashida ${ }^{38}$, S. Axani ${ }^{15}$, X. Bai ${ }^{46}$, A. Balagopal V. ${ }^{38}$, A. Barbano ${ }^{28}$, S. W. Barwick ${ }^{30}$, B. Bastian ${ }^{59}$, V. Basu ${ }^{38}$, S. Baur ${ }^{12}$, R. Bay ${ }^{8}$, J. J. Beatty ${ }^{20,21}$, K.-H. Becker ${ }^{58}$, J. Becker Tjus ${ }^{11}$, C. Bellenghi $^{27}$, S. BenZvi ${ }^{48}$, D. Berley ${ }^{19}$, E. Bernardini ${ }^{59,60}$, D. Z. Besson ${ }^{34,61}$, G. Binder ${ }^{8,9}$, D. Bindig ${ }^{58}$, E. Blaufuss ${ }^{19}$, S. Blot ${ }^{59}$, M. Boddenberg ${ }^{1}$, F. Bontempo ${ }^{31}$, J. Borowka ${ }^{1}$, S. Böser ${ }^{39}$, O. Botner ${ }^{57}$, J. Böttcher ${ }^{1}$, E. Bourbeau ${ }^{22}$, F. Bradascio ${ }^{59}$, J. Braun ${ }^{38}$, S. Bron $^{28}$, J. Brostean-Kaiser ${ }^{59}$, S. Browne ${ }^{32}$, A. Burgman ${ }^{57}$, R. T. Burley ${ }^{2}$, R. S. Busse ${ }^{41}$, M. A. Campana ${ }^{45}$, E. G. Carnie-Bronca ${ }^{2}$, C. Chen ${ }^{6}$, D. Chirkin ${ }^{38}$, K. Choi ${ }^{52}$, B. A. Clark ${ }^{24}$, K. Clark ${ }^{33}$, L. Classen ${ }^{41}$, A. Coleman ${ }^{42}$, G. H. Collin ${ }^{15}$, J. M. Conrad ${ }^{15}$, P. Coppin $^{13}$, P. Correa ${ }^{13}$, D. F. Cowen ${ }^{55,56}$, R. Cross ${ }^{48}$, C. Dappen ${ }^{1}$, P. Dave ${ }^{6}$, C. De Clercq ${ }^{13}$, J. J. DeLaunay ${ }^{56}$, H. Dembinski ${ }^{42}$, K. Deoskar $^{50}$, S. De Ridder ${ }^{29}$, A. Desai ${ }^{38}$, P. Desiati ${ }^{38}$, K. D. de Vries ${ }^{13}$, G. de Wasseige ${ }^{13}$, M. de With ${ }^{10}$, T. DeYoung $^{24}$, S. Dharani $^{1}$, A. Diaz ${ }^{15}$, J. C. Díaz-Vélez ${ }^{38}$, M. Dittmer ${ }^{41}$, H. Dujmovic ${ }^{31}$, M. Dunkman ${ }^{56}$, M. A. DuVernois ${ }^{38}$, E. Dvorak ${ }^{46}$, T. Ehrhardt ${ }^{39}$, P. Eller $^{27}$, R. Engel ${ }^{31,}{ }^{32}$, H. Erpenbeck ${ }^{1}$, J. Evans ${ }^{19}$, P. A. Evenson ${ }^{42}$, K. L. Fan ${ }^{19}$, A. R. Fazely ${ }^{7}$, S. Fiedlschuster ${ }^{26}$, A. T. Fienberg ${ }^{56}$, K. Filimonov ${ }^{8}$, C. Finley ${ }^{50}$, L. Fischer ${ }^{59}$, D. Fox ${ }^{55}$, A. Franckowiak ${ }^{11,59}$, E. Friedman ${ }^{19}$, A. Fritz ${ }^{39}$, P. Fürst ${ }^{1}$, T. K. Gaisser ${ }^{42}$, J. Gallagher $^{37}$, E. Ganster ${ }^{1}$, A. Garcia ${ }^{14}$, S. Garrappa ${ }^{59}$, L. Gerhardt ${ }^{9}$, A. Ghadimi ${ }^{54}$, C. Glaser ${ }^{57}$, T. Glauch ${ }^{27}$, T. Glüsenkamp ${ }^{26}$, A. Goldschmidt $^{9}$, J. G. Gonzalez ${ }^{42}$, S. Goswami ${ }^{54}$, D. Grant ${ }^{24}$, T. Grégoire ${ }^{56}$, S. Griswold ${ }^{48}$, M. Gündüz ${ }^{11}$, C. Günther ${ }^{1}$, C. Haack ${ }^{27}$,

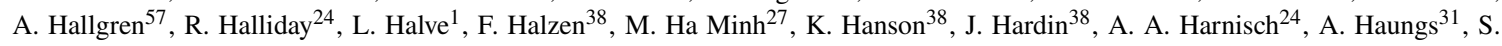

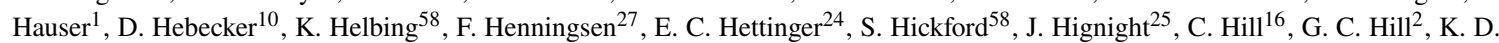

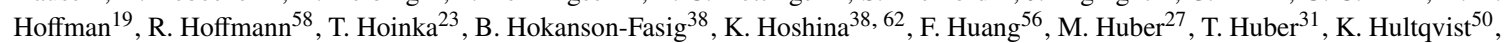
M. Hünnefeld ${ }^{23}$, R. Hussain ${ }^{38}$, S. In ${ }^{52}$, N. Iovine ${ }^{12}$, A. Ishihara ${ }^{16}$, M. Jansson ${ }^{50}$, G. S. Japaridze ${ }^{5}$, M. Jeong ${ }^{52}$, B. J. P. Jones ${ }^{4}$, D. Kang ${ }^{31}$, W. Kang ${ }^{52}$, X. Kang ${ }^{45}$, A. Kappes ${ }^{41}$, D. Kappesser ${ }^{39}$, T. Karg ${ }^{59}$, M. Karl ${ }^{27}$, A. Karle ${ }^{38}$, U. Katz ${ }^{26}$, M. Kauer ${ }^{38}$, M. Kellermann ${ }^{1}$, J. L. Kelley $^{38}$, A. Kheirandish ${ }^{56}$, K. Kin ${ }^{16}$, T. Kintscher ${ }^{59}$, J. Kiryluk ${ }^{51}$, S. R. Klein ${ }^{8,9}$, R. Koirala ${ }^{42}$, H. Kolanoski ${ }^{10}$, T. Kontrimas ${ }^{27}$, L. $\mathrm{Köpke}^{39}$, C. Kopper ${ }^{24}$, S. Kopper ${ }^{54}$, D. J. Koskinen ${ }^{22}$, P. Koundal ${ }^{31}$, M. Kovacevich ${ }^{45}$, M. Kowalski ${ }^{10,59}$, T. Kozynets ${ }^{22}$, E. Kun ${ }^{11}$, N. Kurahashi ${ }^{45}$, N. Lad ${ }^{59}$, C. Lagunas Gualda ${ }^{59}$, J. L. Lanfranchi ${ }^{56}$, M. J. Larson ${ }^{19}$, F. Lauber ${ }^{58}$, J. P. Lazar ${ }^{14,38}$, J. W. Lee ${ }^{52}$, K. Leonard $^{38}$, A. Leszczyńska ${ }^{32}$, Y. Li ${ }^{56}$, M. Lincetto ${ }^{11}$, Q. R. Liu ${ }^{38}$, M. Liubarska ${ }^{25}$, E. Lohfink ${ }^{39}$, C. J. Lozano Mariscal ${ }^{41}$, L. Lu ${ }^{38}$,

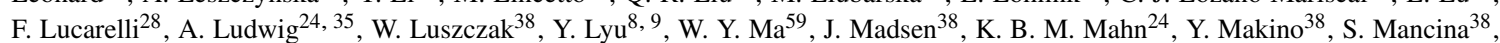

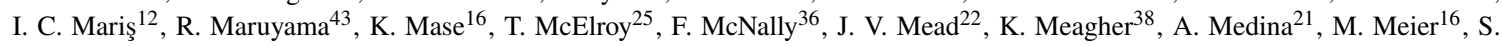
Meighen-Berger ${ }^{27}$, J. Micallef ${ }^{24}$, D. Mockler ${ }^{12}$, T. Montaruli ${ }^{28}$, R. W. Moore ${ }^{25}$, R. Morse ${ }^{38}$, M. Moulai ${ }^{15}$, R. Naab ${ }^{59}$, R. Nagai ${ }^{16}$, U. Naumann ${ }^{58}$, J. Necker ${ }^{59}$, L. V. Nguyễn ${ }^{24}$, H. Niederhausen ${ }^{27}$, M. U. Nisa ${ }^{24}$, S. C. Nowicki ${ }^{24}$, D. R. Nygren ${ }^{9}$, A. Obertacke Pollmann ${ }^{58}$, M. Oehler ${ }^{31}$, A. Olivas ${ }^{19}$, E. O'Sullivan ${ }^{57}$, H. Pandya ${ }^{42}$, D. V. Pankova ${ }^{56}$, N. Park ${ }^{33}$, G. K. Parker ${ }^{4}$, E. N. Paudel ${ }^{42}$, L. Paul ${ }^{40}$, C. Pérez de los $\operatorname{Heros}^{57}$, L. Peters ${ }^{1}$, J. Peterson ${ }^{38}$, S. Philippen ${ }^{1}$, D. Pieloth ${ }^{23}$, S. Pieper ${ }^{58}$, M. Pittermann ${ }^{32}$, A. Pizzuto ${ }^{38}$, M. Plum ${ }^{40}$, Y. Popovych $^{39}$, A. Porcelli ${ }^{29}$, M. Prado Rodriguez ${ }^{38}$, P. B. Price ${ }^{8}$, B. Pries ${ }^{24}$, G. T. Przybylski ${ }^{9}$, C. Raab ${ }^{12}$, A. Raissi ${ }^{18}$, M. Rameez ${ }^{22}$, K. $^{2}$. Rawlins $^{3}$, I. C. Rea ${ }^{27}$, A. Rehman ${ }^{42}$, P. Reichherzer ${ }^{11}$, R. Reimann ${ }^{1}$, G. Renzi ${ }^{12}$, E. Resconi ${ }^{27}$, S. Reusch ${ }^{59}$, W. Rhode ${ }^{23}$, M. Richman ${ }^{45}$, B. Riedel ${ }^{38}$, E. J. Roberts ${ }^{2}$, S. Robertson ${ }^{8,9}$, G. Roellinghoff ${ }^{52}$, M. Rongen ${ }^{39}$, C. Rott ${ }^{49}, 52$, T. Ruhe ${ }^{23}$, D. Ryckbosch ${ }^{29}$, D. Rysewyk $\mathrm{Cantu}^{24}$, I. Safa ${ }^{14,38}$, J. Saffer ${ }^{32}$, S. E. Sanchez Herrera ${ }^{24}$, A. Sandrock ${ }^{23}$, J. Sandroos ${ }^{39}$, M. Santander ${ }^{54}$, S. Sarkar ${ }^{44}$, S. Sarkar ${ }^{25}$, K. Satalecka $^{59}$, M. Scharf ${ }^{1}$, M. Schaufel ${ }^{1}$, H. Schieler ${ }^{31}$, S. Schindler ${ }^{26}$, P. Schlunder ${ }^{23}$, T. Schmidt ${ }^{19}$, A. Schneider ${ }^{38}$, J. Schneider ${ }^{26}$, F. G. Schröder ${ }^{31,42}$, L. Schumacher ${ }^{27}$, G. Schwefer ${ }^{1}$, S. Sclafani ${ }^{45}$, D. Seckel ${ }^{42}$, S. Seunarine ${ }^{47}$, A. Sharma ${ }^{57}$, S. Shefali ${ }^{32}$, M. Silva $^{38}$, B. Skrzypek ${ }^{14}$, B. Smithers ${ }^{4}$, R. Snihur ${ }^{38}$, J. Soedingrekso ${ }^{23}$, D. Soldin ${ }^{42}$, C. Spannfellner ${ }^{27}$, G. M. Spiczak ${ }^{47}$, C. Spiering ${ }^{59,}$, ${ }^{2}$, J. Stachurska $^{59}$, M. Stamatikos ${ }^{21}$, T. Stanev ${ }^{42}$, R. Stein ${ }^{59}$, J. Stettner ${ }^{1}$, A. Steuer ${ }^{39}$, T. Stezelberger ${ }^{9}$, T. Stürwald ${ }^{58}$, T. Stuttard ${ }^{22}$, G. W. Sullivan $^{19}$, I. Taboada ${ }^{6}$, F. Tenholt ${ }^{11}$, S. Ter-Antonyan ${ }^{7}$, S. Tilav ${ }^{42}$, F. Tischbein ${ }^{1}$, K. Tollefson $^{24}$, L. Tomankova ${ }^{11}$, C. Tönnis ${ }^{53}$, S. Toscano $^{12}$, D. Tosi $^{38}$, A. Trettin ${ }^{59}$, M. Tselengidou ${ }^{26}$, C. F. Tung ${ }^{6}$, A. Turcati ${ }^{27}$, R. Turcotte ${ }^{31}$, C. F. Turley ${ }^{56}$, J. P. Twagirayezu ${ }^{24}$, B. $\mathrm{Ty}^{38}$, M. A. Unland Elorrieta ${ }^{41}$, N. Valtonen-Mattila ${ }^{57}$, J. Vandenbroucke ${ }^{38}$, N. van Eijndhoven ${ }^{13}$, D. Vannerom ${ }^{15}$, J. van Santen $^{59}, \mathrm{~S}$.

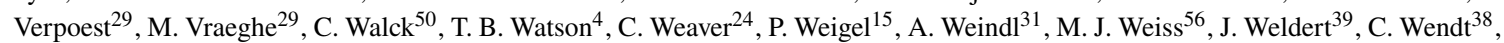
J. Werthebach ${ }^{23}$, M. Weyrauch ${ }^{32}$, N. Whitehorn ${ }^{24,35}$, C. H. Wiebusch ${ }^{1}$, D. R. Williams ${ }^{54}$, M. Wolf ${ }^{27}$, K. Woschnagg ${ }^{8}$, G. Wrede ${ }^{26}$, J.

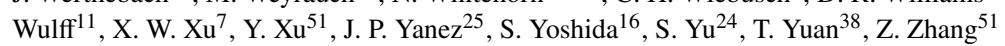

${ }^{1}$ III. Physikalisches Institut, RWTH Aachen University, D-52056 Aachen, Germany

2 Department of Physics, University of Adelaide, Adelaide, 5005, Australia

${ }^{3}$ Dept. of Physics and Astronomy, University of Alaska Anchorage, 3211 Providence Dr., Anchorage, AK 99508, USA

${ }^{4}$ Dept. of Physics, University of Texas at Arlington, 502 Yates St., Science Hall Rm 108, Box 19059, Arlington, TX 76019, USA

5 CTSPS, Clark-Atlanta University, Atlanta, GA 30314, USA

${ }^{6}$ School of Physics and Center for Relativistic Astrophysics, Georgia Institute of Technology, Atlanta, GA 30332, USA

${ }^{7}$ Dept. of Physics, Southern University, Baton Rouge, LA 70813, USA

${ }^{8}$ Dept. of Physics, University of California, Berkeley, CA 94720, USA

${ }^{9}$ Lawrence Berkeley National Laboratory, Berkeley, CA 94720, USA

${ }^{10}$ Institut für Physik, Humboldt-Universität zu Berlin, D-12489 Berlin, Germany

${ }^{11}$ Fakultät für Physik \& Astronomie, Ruhr-Universität Bochum, D-44780 Bochum, Germany

12 Université Libre de Bruxelles, Science Faculty CP230, B-1050 Brussels, Belgium

13 Vrije Universiteit Brussel (VUB), Dienst ELEM, B-1050 Brussels, Belgium

14 Department of Physics and Laboratory for Particle Physics and Cosmology, Harvard University, Cambridge, MA 02138, USA

15 Dept. of Physics, Massachusetts Institute of Technology, Cambridge, MA 02139, USA 
16 Dept. of Physics and Institute for Global Prominent Research, Chiba University, Chiba 263-8522, Japan

17 Department of Physics, Loyola University Chicago, Chicago, IL 60660, USA

18 Dept. of Physics and Astronomy, University of Canterbury, Private Bag 4800, Christchurch, New Zealand

19 Dept. of Physics, University of Maryland, College Park, MD 20742, USA

${ }^{20}$ Dept. of Astronomy, Ohio State University, Columbus, OH 43210, USA

${ }^{21}$ Dept. of Physics and Center for Cosmology and Astro-Particle Physics, Ohio State University, Columbus, OH 43210, USA

22 Niels Bohr Institute, University of Copenhagen, DK-2100 Copenhagen, Denmark

23 Dept. of Physics, TU Dortmund University, D-44221 Dortmund, Germany

${ }^{24}$ Dept. of Physics and Astronomy, Michigan State University, East Lansing, MI 48824, USA

25 Dept. of Physics, University of Alberta, Edmonton, Alberta, Canada T6G 2E1

${ }^{26}$ Erlangen Centre for Astroparticle Physics, Friedrich-Alexander-Universität Erlangen-Nürnberg, D-91058 Erlangen, Germany

27 Physik-department, Technische Universität München, D-85748 Garching, Germany

28 Département de physique nucléaire et corpusculaire, Université de Genève, CH-1211 Genève, Switzerland

${ }^{29}$ Dept. of Physics and Astronomy, University of Gent, B-9000 Gent, Belgium

${ }^{30}$ Dept. of Physics and Astronomy, University of California, Irvine, CA 92697, USA

${ }^{31}$ Karlsruhe Institute of Technology, Institute for Astroparticle Physics, D-76021 Karlsruhe, Germany

${ }^{32}$ Karlsruhe Institute of Technology, Institute of Experimental Particle Physics, D-76021 Karlsruhe, Germany

33 Dept. of Physics, Engineering Physics, and Astronomy, Queen's University, Kingston, ON K7L 3N6, Canada

34 Dept. of Physics and Astronomy, University of Kansas, Lawrence, KS 66045, USA

35 Department of Physics and Astronomy, UCLA, Los Angeles, CA 90095, USA

36 Department of Physics, Mercer University, Macon, GA 31207-0001, USA

${ }^{37}$ Dept. of Astronomy, University of Wisconsin-Madison, Madison, WI 53706, USA

${ }^{38}$ Dept. of Physics and Wisconsin IceCube Particle Astrophysics Center, University of Wisconsin-Madison, Madison, WI 53706, USA

${ }^{39}$ Institute of Physics, University of Mainz, Staudinger Weg 7, D-55099 Mainz, Germany

40 Department of Physics, Marquette University, Milwaukee, WI, 53201, USA

${ }^{41}$ Institut für Kernphysik, Westfälische Wilhelms-Universität Münster, D-48149 Münster, Germany

42 Bartol Research Institute and Dept. of Physics and Astronomy, University of Delaware, Newark, DE 19716, USA

43 Dept. of Physics, Yale University, New Haven, CT 06520, USA

${ }^{44}$ Dept. of Physics, University of Oxford, Parks Road, Oxford OX1 3PU, UK

45 Dept. of Physics, Drexel University, 3141 Chestnut Street, Philadelphia, PA 19104, USA

46 Physics Department, South Dakota School of Mines and Technology, Rapid City, SD 57701, USA

47 Dept. of Physics, University of Wisconsin, River Falls, WI 54022, USA

48 Dept. of Physics and Astronomy, University of Rochester, Rochester, NY 14627, USA

49 Department of Physics and Astronomy, University of Utah, Salt Lake City, UT 84112, USA

50 Oskar Klein Centre and Dept. of Physics, Stockholm University, SE-10691 Stockholm, Sweden

${ }^{51}$ Dept. of Physics and Astronomy, Stony Brook University, Stony Brook, NY 11794-3800, USA

52 Dept. of Physics, Sungkyunkwan University, Suwon 16419, Korea

53 Institute of Basic Science, Sungkyunkwan University, Suwon 16419, Korea

54 Dept. of Physics and Astronomy, University of Alabama, Tuscaloosa, AL 35487, USA

55 Dept. of Astronomy and Astrophysics, Pennsylvania State University, University Park, PA 16802, USA

56 Dept. of Physics, Pennsylvania State University, University Park, PA 16802, USA

${ }^{57}$ Dept. of Physics and Astronomy, Uppsala University, Box 516, S-75120 Uppsala, Sweden

58 Dept. of Physics, University of Wuppertal, D-42119 Wuppertal, Germany

59 DESY, D-15738 Zeuthen, Germany

${ }^{60}$ Università di Padova, I-35131 Padova, Italy

61 National Research Nuclear University, Moscow Engineering Physics Institute (MEPhI), Moscow 115409, Russia

${ }^{62}$ Earthquake Research Institute, University of Tokyo, Bunkyo, Tokyo 113-0032, Japan

\section{Acknowledgements}

USA - U.S. National Science Foundation-Office of Polar Programs, U.S. National Science Foundation-Physics Division, U.S. National Science Foundation-EPSCoR, Wisconsin Alumni Research Foundation, Center for High Throughput Computing (CHTC) at the University of Wisconsin-Madison, Open Science Grid (OSG), Extreme Science and Engineering Discovery Environment (XSEDE), Frontera computing project at the Texas Advanced Computing Center, U.S. Department of Energy-National Energy Research Scientific Computing Center, Particle astrophysics research computing center at the University of Maryland, Institute for Cyber-Enabled Research at Michigan State University, and Astroparticle physics computational facility at Marquette University; Belgium - Funds for Scientific Research (FRS-FNRS and FWO), FWO Odysseus and Big Science programmes, and Belgian Federal Science Policy Office (Belspo); Germany - Bundesministerium für Bildung und Forschung (BMBF), Deutsche Forschungsgemeinschaft (DFG), Helmholtz Alliance for Astroparticle Physics (HAP), Initiative and Networking Fund of the Helmholtz Association, Deutsches Elektronen Synchrotron (DESY), and High Performance Computing cluster of the RWTH Aachen; Sweden - Swedish Research Council, Swedish Polar Research Secretariat, Swedish National Infrastructure for Computing (SNIC), and Knut and Alice Wallenberg Foundation; Australia - Australian 
Research Council; Canada - Natural Sciences and Engineering Research Council of Canada, Calcul Québec, Compute Ontario, Canada Foundation for Innovation, WestGrid, and Compute Canada; Denmark - Villum Fonden and Carlsberg Foundation; New Zealand Marsden Fund; Japan - Japan Society for Promotion of Science (JSPS) and Institute for Global Prominent Research (IGPR) of Chiba University; Korea - National Research Foundation of Korea (NRF); Switzerland - Swiss National Science Foundation (SNSF); United Kingdom - Department of Physics, University of Oxford. 